\title{
Propagation-based X-ray Phase Contrast Microtomography of Zebrafish Embryos to Understand Drug Delivery
}

\author{
G. Schulz $^{1, *}$, E. Cörek ${ }^{2}$, S. Siegrist ${ }^{2}$, P. Thalmann ${ }^{1}$, H. Deyhle ${ }^{1}$, A. Rack ${ }^{3}$, J. Huwyler ${ }^{2}$, and B. Müller ${ }^{1}$
}

1. University of Basel, Allschwil, Switzerland.

2. University of Basel, Basel, Switzerland.

3. ESRF - The European Synchrotron, Grenoble, France.

* Corresponding author, georg.schulz@unibas.ch

The zebrafish embryo has been widely discussed as an in vivo screening model for nanotoxicology [1,2], mainly for studying host-pathogen interactions, innate immune responses, and inflammation. The vertebrate model is convenient because of its transparency, rapid life cycle, a high similarity of the innate immune system to humans, easy husbandry, and size, i.e. 0.5 to $0.7 \mathrm{~mm}$ in diameter and 5 to $7 \mathrm{~mm}$ long. Furthermore, no animal permission is needed up to 120 hours post fertilization. Nowadays, the investigations of the zebrafish embryos are mainly based on visible light and confocal microscopy. Nanoparticles are widely used to investigate a plethora of phenomena. For the localization of the nanoparticle distribution within the embryo, a non-destructive 3D method is beneficial. Conventional hard X-ray microtomography shows marginal contrast within soft tissues. Having a resolution below the wavelength of visible light [3], adequate contrast within soft tissues and reasonable scan times as required for imaging large numbers of specimens, single-distance phase contrast (SDPC) microtomography using ANKAphase phase retrieval $[4,5]$ was chosen for our study.

Zebrafish embryos were euthanized 48 hours post fertilization and one hour post nanoparticle injection, fixed in $4.0 \%$ paraformaldehyde (PFA), and dehydrated in an increasing ethanol dilution series. The dehydrated zebrafish embryo was stored in $100 \%$ ethanol at atemperature of $4.0{ }^{\circ} \mathrm{C}$. After air-drying, the embryo was placed into an aluminum mold channel. The mold was immersed vertically into liquid paraffin wax allowing filling with paraffin due to capillary forces. After cooling, the mold was opened and the paraffin rod containing the embedded embryo was removed. Intravenous microinjections of e.g. nanoparticles was done into the Duct of Cuvier which is a temporary circulation channel until 72 hours post fertilization. The SDPC measurements were carried out at the beamline ID19 (ESRF, Grenoble, France) with a mean photon energy of $19.6 \mathrm{keV}$ at a sample-detector distance of $10 \mathrm{~mm}$. For the detection, a pco.5.5 camera (PCO AG, Kelheim, Germany) with an effective pixel size of $0.3 \mu \mathrm{m}$ was applied. Exposure time per radiograph was set to $0.1 \mathrm{~s}$, resulting in a total acquisition time of around 15 minutes per height step. Using asymmetric rotation axis position, 5120 projections were recorded over $360^{\circ}$. Due to the field of view of $0.83 \times 0.70 \mathrm{~mm}^{2}$, four height steps were necessary to image the entire zebrafish embryo. The phase retrieval was performed in ANKAphase [4] with a $\delta / \beta$ ratio of 1,000 . The phase projections were reconstructed using a standard filtered back-projection algorithm implemented in MATLAB R2017b (Simulink, The MathWorks, Inc., USA). For the visualization of the tomograms VGStudio MAX 2.2 (Volume Graphics GmbH, Heidelberg, Germany) was used.

The 3D rendering of the reconstructed data set in Figure 1 (a) shows zebrafish embryo anatomy. SDPC is sufficient to reveal the smallest structures e.g. the eye lens measuring around $35 \mu \mathrm{m}$ in diameter (Figure 1 (b)). After making the soft tissue virtually transparent (Figure 1 (c)), otoliths, which are stony accretions consisting of crystalline calcium carbonate and protein in the ear of the embryo [6], could be uncovered. The otoliths are also illustrated in the selected virtual cut through the data set (Figure 1 (d)). In addition, the reconstructed slice demonstrates the high contrast of SDPC showing differences within soft tissue. 
SDPC microtomography with a sub-cellular resolution allows the non-destructive visualization of paraffin-embedded zebrafish embryos without the use of any contrast agent. It detects electron density differences within the soft tissues and simultaneously visualizes soft and hard tissues (otoliths). Both spatial resolution and contrast are sufficient to investigate the 3D distribution of injected nanoparticles.

\section{References:}

[1] H Fischer et al. Current Opinion in Biotechnology 18 (2007), 565-571

[2] Z Clemente et al. Science of the Total Environment 463 (2013), 647-656

[3] A Khimchenko et al, Advanced Science (2018), 1700694

[4] T Weitkamp et al, Journal of Synchrotron Radiation 18 (2011), 617

[5] D Paganin et al, Journal of Microscopy 206 (2002), 33

[6] B Müller et al, International Journal of Materials Research 98 (2007), 613-621

[6] The project was supported by allocation of beamtime at ESRF (MD1017). The financial contribution of Swiss National Science Foundation project 144535 and R'Equip project 133802 is acknowledged.

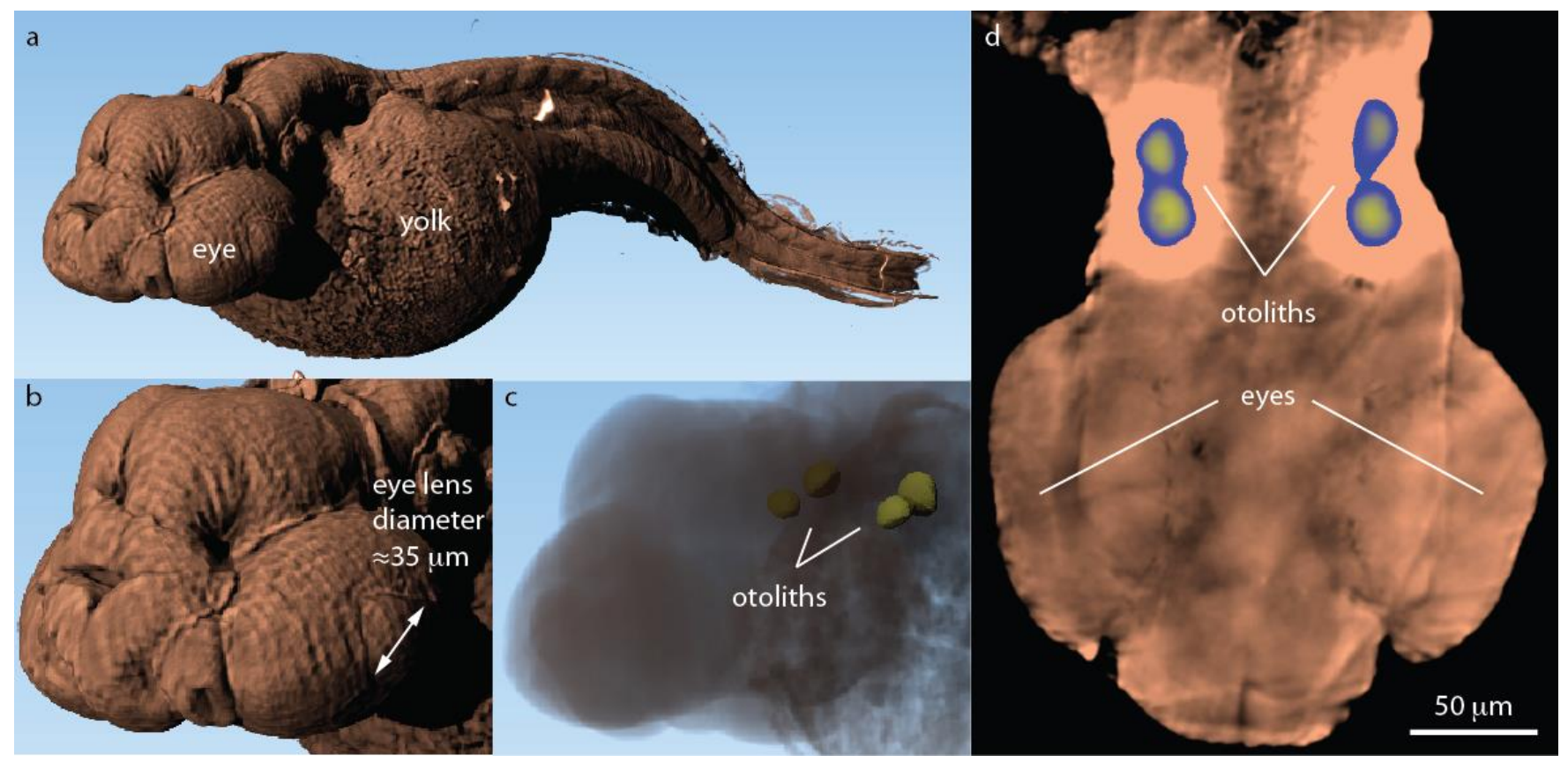

Figure. 1. 3D rendering of the entire fish embryo (a) and a zoom of the head reveal external structures of the zebra fish embryo. Transparent representation of the zoom in (c) uncovers internal features of the specimen. A selected virtual cut through the head of the specimens (d) shows the internal microstructures. 